\title{
Video Article \\ Determining and Controlling External Power Output During Regular Handrim Wheelchair Propulsion
}

\author{
Rick de Klerk ${ }^{1}$, Riemer J. K. Vegter ${ }^{1}$, Marika T. Leving ${ }^{1}$, Sonja de Groot ${ }^{1,2}$, DirkJan H. E. J. Veeger ${ }^{3,4}$, Lucas H. V. van der Woude ${ }^{1,5}$ \\ ${ }^{1}$ Center for Human Movement Sciences, University Medical Center Groningen \\ ${ }^{2}$ Amsterdam Rehabilitation Research Center Reade, Amsterdam \\ ${ }^{3}$ Research Institute MOVE, Faculty of Behavioral and Human Movement Sciences, VU University \\ ${ }^{4} 3 \mathrm{M}$, Delft University of Technology, Delft \\ ${ }^{5}$ Center for Rehabilitation, University Medical Center Groningen
}

Correspondence to: Rick de Klerk at r.de.klerk@umcg.nl

URL: https://www.jove.com/video/60492

DOI: doi:10.3791/60492

Keywords: Medicine, Issue 156, wheelchairs, propulsion technique, motor skills, efficiency, ergonomics, ergometry, biomechanical phenomena

Date Published: 2/5/2020

Citation: de Klerk, R., Vegter, R.J.K., Leving, M.T., de Groot, S., Veeger, D.H.E.J., van der Woude, L.H.V. Determining and Controlling External Power Output During Regular Handrim Wheelchair Propulsion. J. Vis. Exp. (156), e60492, doi:10.3791/60492 (2020).

\section{Abstract}

The use of a manual wheelchair is critical to $1 \%$ of the world's population. Human powered wheeled mobility research has considerably matured, which has led to improved research techniques becoming available over the last decades. To increase the understanding of wheeled mobility performance, monitoring, training, skill acquisition, and optimization of the wheelchair-user interface in rehabilitation, daily life, and sports, further standardization of measurement set-ups and analyses is required. A crucial stepping-stone is the accurate measurement and standardization of external power output (measured in Watts), which is pivotal for the interpretation and comparison of experiments aiming to improve rehabilitation practice, activities of daily living, and adaptive sports. The different methodologies and advantages of accurate power output determination during overground, treadmill, and ergometer-based testing are presented and discussed in detail. Overground propulsion provides the most externally valid mode for testing, but standardization can be troublesome. Treadmill propulsion is mechanically similar to overground propulsion, but turning and accelerating is not possible. An ergometer is the most constrained and standardization is relatively easy. The goal is to stimulate good practice and standardization to facilitate the further development of theory and its application among research facilities and applied clinical and sports sciences around the world.

\section{Video Link}

The video component of this article can be found at https://www.jove.com/video/60492/

\section{Introduction}

With an estimated $1 \%$ of the world's population being dependent on wheeled mobility today ${ }^{1,2}$, a consistent flow of international research work increasingly emerges into international peer-reviewed journals in diverse fields such as rehabilitation ${ }^{1,3}$, engineering ${ }^{4}$, and sport sciences ${ }^{5,6}$. This leads to a growing knowledge base and understanding of the complexities of this common mode of human ambulation. Yet, for continual development and implementation in rehabilitation and adaptive sport practices, there is a need for further international exchange and collaboration in research. Integral to such collaborative networks are improved standardization of experimental and measurement procedures and technology. Furthermore, consistent implementation of accurate monitoring of performance of the wheelchair-user combination in the laboratory and/or in the field is important for an optimal individual functioning and participation while a healthy and active lifestyle is maintained over the individual's lifespan ${ }^{7,8,9}$.

Experimentally, manual wheelchair propulsion during steady-state or peak exercise conditions ${ }^{10,11}$ is often approached as cyclical upper body motion for the purposes of examining the wheelchair-user interface ${ }^{12,13}$, musculoskeletal loading ${ }^{14,15,16}$, and motor learning and skill acquisition ${ }^{17,18}$. The combined biomechanical and physiological notions of cyclic motions allow the use of the "Power balance", a modelling approach that was initially introduced by Van Ingen Schenau ${ }^{19}$ for speed skating and cycling, and later introduced in manual wheeled mobility $8,20,21$. Figure 1 shows a power balance diagram for manual wheelchair propulsion. It converges from a selection of critical performance determining factors for the wheelchair-user combination and its three central components (the wheelchair, user, and their interface), at the lefthand side into the layout of (bio)mechanical and physiological power denominators and equations.

Power output is an important outcome parameter in the contexts of sports and daily life where peak power output can represent both increased performance in adapted sports or ease of functioning during activities in daily living ${ }^{22}$. Moreover, in combination with energy consumption it can be used to evaluate performance in terms of gross mechanical efficiency ${ }^{17,18,23}$ (i.e., where a more skilled individual would require less internal energy to produce the same amount of external power output). From an experimental perspective, power output is a parameter that needs to be tightly controlled during a test, because changes in power output are of direct influence on all performance outcomes such as push time, 
recovery time ${ }^{24}$, and mechanical efficiency ${ }^{25}$. Consequently, controlling and reporting power output is essential for all studies related to manual wheelchair propulsion.

Overground testing is the gold standard in terms of validity (i.e., inertia, air friction, optical flow, and dynamic movement) ${ }^{26}$, yet standardization of external power output, speed, and associated environmental conditions is much more difficult, and repeatability over time suffers. Overground wheelchair-related studies started in the $1960 \mathrm{~s}^{27,28}$ and focused on the physical strain of wheeled mobility. Although crucial in data interpretation and understanding ${ }^{8,20}$, notions on external power output were limited to observation of the internal metabolic cost when performing different activities on different surfaces. Nowadays, measurement wheels can be used to measure power output ${ }^{29,30}$ and coast-down tests ${ }^{31,32}$ can be $^{2}$ performed to infer the frictional losses during propulsion and thereby power output.

Different laboratory-based technologies were developed for wheelchair-specific exercise testing ${ }^{33}$, ranging from a multitude of ergometers to differently sized and brands of treadmills. Treadmills are considered to be closest to overground testing in terms of validity ${ }^{34}$ and have been used since the 1960 s for wheelchair exercise testing ${ }^{35,36}$. Prior to testing, the slope and speed of the treadmill must be checked regularly. Even treadmills from the same brand and make may differ considerably and change in their behavior over time ${ }^{37}$. For the determination of external power output, a drag test ${ }^{20,36}$ is used for the individual wheelchair-user combination's total of rolling and internal drag force ${ }^{38}$. The force sensor for the drag test also has to be periodically calibrated. For the experimental individualization of the protocol in terms of overall external load of wheeling over time and between subjects, a pulley system (Figure 2) has been designed as an alternative for the previous slope-dependent gradients of loading ${ }^{36}$.

Another alternative for standardized wheelchair exercise testing has been the use of stationary ergometers ${ }^{33}$, from simple off-the shelf ergometer solutions ${ }^{39}$ towards highly specialized computer-based and instrumented ergometers ${ }^{40}$. Very few are commercially available. The enormous diversity in ergometer technology and mechanical characteristics introduces large unknown degrees of variability among the test outcomes ${ }^{33}$. Ergometers and wheelchairs need to be connected or inherently fused by design. Air friction is not present and perceived inertia is limited to the simulated inertia on the wheels, and movement experienced in the trunk, head, and arms during propulsion, while the wheelchair user is essentially stationary. The ergometer does allow for sprint or anaerobic testing as well as isometric testing, if the wheels can be adequately blocked.

A basic methodology for manual wheeled mobility research in lab-based studies is presented. Also, a brief outlook on field-based wheelchair research methodology and its potential outcomes is provided. The central focus is controlling and measuring external power output (W) in both field and laboratory-based experiments. The determination of internal power output through spirometry is also added, as this is often used to determine gross mechanical efficiency. Apart from the implementation of good practice, the goal is to produce discussions on experimental standardization and international information exchange. The current study will primarily address handrim wheelchair propulsion and the measurement thereof because it is the most prominent form of manually wheeled mobility in scientific literature. However, notions discussed below are equally valid for other wheelchair propulsion mechanisms (e.g., levers, cranks ${ }^{41}$ ).

The current protocol describes the standardization and measurement of power output during overground, treadmill, and wheelchair ergometerbased testing during steady-state propulsion at $1.11 \mathrm{~m} / \mathrm{s}$. As an example, rolling friction will first be determined in overground testing with a coast-down test. Using this estimate of friction, power outputs will be set in the treadmill and ergometer tests using available protocols from the research literature. For treadmill tests, friction will be determined with a drag test, and power output will be adjusted using a pulley system. For the ergometer tests a computer-controlled ergometer is used to match external power output with the overground test.

\section{Protocol}

This study was approved by the local ethical committee (Ethical Committee Human Movement Sciences) at the University Medical Center Groningen. All participants signed written informed consent.

\section{Study design and setup}

1. Instruct the participant and obtain informed consent in line with the Ethical Committee of the institution.

2. Determine the readiness for physical activity of the participants by performing a baseline assessment with the Physical Activity Readiness Questionnaire ${ }^{42,43}$.

3. Perform in-patient screening with a medical doctor

4. Decide on a fixed power output for all participants (e.g., $10-20 \mathrm{~W}$ at $1.11 \mathrm{~m} / \mathrm{s}$ ), a relative power output (e.g., $0.25 \mathrm{~W} / \mathrm{kg}$ bodyweight at $1.11 \mathrm{~m} /$ s), or a "realistic" individual power output based on a surface of interest (based on a coast-down test).

5. Allow the participant to become familiar with the overground, treadmill, and ergometer conditions before testing.

6. Check the tire pressure and overall wheelchair mechanics before each measurement and inflate the tires to $600 \mathrm{kPa}$ if needed. NOTE: To obtain valid steady-state outcomes for cardiopulmonary upper body work and gross mechanical efficiency (ME), one should adhere to a minimum duration of 3 min per submaximal (up to $70 \%$ peak exercise capacity) exercise block to achieve steady-state exercise with a respiratory exchange ratio below $1^{44,45}$. Especially in handrim propulsion, the wheelchair speed must remain within a comfortable or feasible range $(0.56-2.0 \mathrm{~m} / \mathrm{s})$ to exclude motor control issues ${ }^{46,47,48}$, which means that power increments are preferably controlled by increments in resistance.

\section{External power output during overground testing}

1. Perform the coast-down test on the surface of interest. Position the participant in an active position and as standardized as possible: feet on the footrest, hands on the lap, and looking straight ahead (the position should be reflective of the position during propulsion).

NOTE: Every movement changes the center of mass, which changes the rolling resistance.

2. Accelerate the wheelchair to a high velocity. 
NOTE: This can also be done by the participant.

3. Let the wheelchair decelerate to a complete standstill without interference.

4. Record the time and velocity data during the deceleration (e.g., with measurement wheels or inertial measurement units). See sections 2.4 .1 and 2.4.2.

1. Record data using measurement wheels.

1. Replace the wheels of the wheelchair with a measurement wheel and the inertial dummy (Table of Materials), preferably while the participant is not in the wheelchair.

NOTE: This example is for the OptiPush wheel. Other wheels might have different calibration requirements.

2. Turn on the measurement wheel using the on/off switch.

3. Turn on the laptop with the USB Bluetooth receiver and associated software.

4. Open the software on the computer.

5. Connect the wheel with the software by selecting the correct communication port (COM). If the correct COM port does not appear in the list, press refresh to update the list and try again. Press Next.

6. Fill in the required fields on the Client Setup screen. Press Next. NOTE: Pay special attention to the Wheel Size and Wheel Side settings.

7. Gather offset data by pressing Start in the wheel setup and slowly spinning the wheel without touching the handrim until the red circle turns to green. Alternatively, press Skip to skip this step if the procedure has already been performed since the last wheel installation. Press Next.

8. To collect data press Record in the Data Collection screen. Resume the regular coast-down protocol from here on. NOTE: Scripts for the analysis of measurement wheel data are available in the Supplementary Material 1.

2. Record the data using inertial measurement units (IMUs).

1. Attach the IMUs (Table of Materials) to the wheelchair: one on each wheel hub and one in the center underneath the seat. Write down which IMU is attached where and in what orientation for later reference.

2. Turn on the IMUs and connect the IMUs to the computer using the NGIMU Synchronized Network Manager executable.

3. To collect data, go to Tools, then select Data Logger, and press Start. Resume the regular coast-down protocol from here on. NOTE: Scripts for the analysis of IMU data are available in the Supplementary Material 2.

5. Repeat the coast-down procedure (2.1-2.4) and collect back-and-forth data to reduce the influence of uneven surfaces.

6. Open the coast_down_test software on a computer. Press Import data to import the coast-down data file (measurement wheel or IMU). Select coast-down sections in the data by using the slider in the graph on the right and pressing Grab selection.

7. Set participant and wheelchair weight in the Settings section. Press Calculate outcomes. Write down the mean rolling friction (N) and rolling friction coefficient. Press Export to save all (meta)data for later reference.

NOTE: When constant friction cannot be assumed due to air drag (i.e., in most sports environments) the analysis becomes a little more complex. The protocol is identical, but the initial speed should probably be higher. In this case, a non-linear differential equation needs to be solved and that equation needs to be fit with a curve fitter (e.g., Levenberg-Marquardt) ${ }^{49}$.

$v_{t}=\sqrt{\frac{c_{2}}{c_{1}}} \tan \left[\tan ^{-1}\left(v_{0} \sqrt{\frac{c_{1}}{c_{2}}}-t \frac{\sqrt{c_{1} c_{2}}}{M}\right)\right]$

In this equation is the instantaneous velocity and is the initial velocity at the start of deceleration. reflects the velocity dependent friction and reflects the velocity independent friction (). Scripts for the analysis of coast-down tests are available in the Supplementary Material $\mathbf{3}$ and the graphical user interface (GUI) for the analysis of coast-down tests used in step 2.7 is available in the Supplementary Material 4.

\section{External power output during treadmill testing}

1. Treadmill characterization

1. Measure the belt velocity of the loaded treadmill with a calibrated tachometer to determine what treadmill setting needs to be used (e.g., for $1.11 \mathrm{~m} / \mathrm{s}$ the treadmill needs to be set to $4.1 \mathrm{~km} / \mathrm{h}$ on the display instead of $4.0 \mathrm{~km} / \mathrm{h}$ ).

NOTE: Alternatively, determine the belt velocity by measuring the belt length and counting ten rotations while recording the time with a stopwatch/video camera.

2. Measure the treadmill angles using an angle sensor. Check for consistency by repeating the measurements and check for hysteresis by repeating the measurements in descending order.

NOTE: Check treadmill velocity with a tachometer and angles with an angle sensor during every measurement if their reliability is low.

2. Drag test: calibration

1. Turn on the power supply of the drag test force sensor at least $30 \mathrm{~min}$ before calibration.

2. Suspend the force transducer vertically and align with a self-levelling laser or angle sensor.

3. Set up the drag test computer and connect the force sensor with the computer. Open the ADA3 software on the drag test computer and press Calibrate force sensor.

4. Attach known (calibrated) weights (1-10 kg with $1 \mathrm{~kg}$ increments) to the sensor and register the digital values.

5. Fit a linear regression equation to determine the relation between applied load and measured voltage by continuing in the ADA3 software.

6. Repeat (3.2.1-3.2.5) if the root-mean-square error (RMSE) exceeds $0.13 \mathrm{~N}^{37}$.

3. Performing a drag test

1. Turn on the power supply at least 30 min before measuring.

2. Set up the drag test computer and connect the force sensor with the computer. Open the ADA3 software on the drag test computer and press Power table measurements. 
3. Place the wheelchair-user combination on the treadmill. Position the participant in an active position and as standardized as possible: feet on the footrest, hands on the lap, and looking straight ahead (the position should be reflective of the position during propulsion). Instruct the participant to maintain the same position throughout the test.

4. Measure the offset of the load cell by recording force with no rope attached using the ADA3 software. Press OK.

5. Connect the wheelchair to the force transducer with a lightweight rope. Ensure the load cell and rope are horizontally aligned with the rear wheel axle of the wheelchair.

6. Accelerate the belt to the desired speed, in this case $1.11 \mathrm{~m} / \mathrm{s}(4.1 \mathrm{~km} / \mathrm{h}$ on display).

7. Increase the inclination of the treadmill, wait until the position of the treadmill and wheelchair-user combination is stable, and record the force and angle. Repeat for 10 increasingly steep angles (1.5-6\% in $0.5 \%$ increments).

8. Fit a linear regression using the angle and force using the ADA3 software by clicking Next. Calculate the force at the zero-angle of the treadmill.

NOTE: The intercept of the regression equation cannot be used if the treadmill angle has an offset.

9. Repeat the drag test (3.3.3-3.3.8) if the RMSE of the linear regression line exceeds $0.5 \mathrm{~N}^{37}$.

4. Setting power output on a treadmill

1. Calculate the desired power output and determine the testing speed.

NOTE: For the current protocol, this is equal to the results obtained in step 2.7.

2. Calculate the required pulley weight by subtracting the drag test friction (from step 3.3.8) from the target friction (from step 2.7).

3. Position the pulley in front or behind the treadmill and make sure it is centered. Attach the pulley to the wheelchair and make sure the rope is level. Instruct the participant that the weight in the pulley might move the wheelchair.

4. Attach the weight (usually between $0-1 \mathrm{~kg}$ ) to the pulley system using a basket of known low mass and a carabiner. Slowly increase the weight if necessary, until the desired power output is achieved.

NOTE: Alternatively change the power output by changing the angle of the treadmill based on the power table from a drag test.

\section{External power output during ergometer-based testing}

1. Turn on the ergometer at least 30 min before measuring. Start the associated software on the computer.

2. Press the Participant widget, then press Add.... Give the participant an ID and enter the body weight of the participant. Press OK.

3. Press the wheelchair icon on the Device menu. Fill in the wheelchair specifications in the form. Press OK. NOTE: The body weight variable is important, because it will influence the simulation provided by the ergometer.

4. Press the protocol widget. Create a custom protocol by selecting Add.... Select Custom Protocol and press Next. Give the protocol an appropriate name and press Create.

5. Select Stages and click Add Stage and Resistance. Set the resistance to the friction coefficient obtained with the coast-down test in section 2 . Set the target speed at $4 \mathrm{~km} / \mathrm{h}$ and press OK (Figure 3 ).

6. Set up the participant screen. Remove all widgets from the screen. Click Add widget and select the Wheelchair direction widget and drag it into the screen (Figure 4).

7. Align the wheelchair on the rollers using the alignment system. Fasten the wheelchair using the four-belt system. Check that the wheels are not touching the ergometer and are properly aligned.

8. Position the participant in an active position and as standardized as possible: feet on the footrest, hands on the lap, and looking straight ahead (the position should be reflective of the position during propulsion). Instruct the participant to maintain the same position throughout the test.

9. Calibrate the ergometer with the associated software by pressing the Crosshair button in the Device menu, and press Start calibration. NOTE: Scripts for the analysis of ergometer data are available in the Supplementary Material 5.

\section{Internal estimates of power output during hand rim wheelchair propulsion}

1. Turn on the spirometer for at least 45 min before any calibrations or testing.

2. Calibrate the spirometer according to the factory guidelines using the associated software, including calibrations for turbine, reference gas, room air, and delay.

NOTE: Room air and reference gas calibrations should be performed before each test.

1. Perform a turbine calibration.

1. Press Turbine in the calibration menu. Connect the turbine with an optoelectronic reader to the spirometer. Connect the calibration syringe with a known volume to the turbine.

2. When the unit is ready, perform six controlled and complete strokes with the piston. Press the Exit icon.

2. Perform a reference gas calibration.

1. Press Reference gas in the calibration menu. Connect the pressure regulator to the calibration cylinder with a known concentration of mixed gas.

NOTE: The cylinder must be open, but the pressure regulator must be closed.

2. Connect the sampling line to the sampling connector of the spirometer and leave the other end disconnected. Let the spirometer flush the analyzers. Make sure that the sampling line is far from any exhaled gas.

3. When prompted by the spirometer, connect the free end of the sampling line to the pressure regulator on the calibration cylinder and open the regulator. Exit with the Exit icon after the calibration ends.

3. Perform a room air calibration.

1. Connect the sampling line to the sampling connector on the spirometer and leave the other end free. Exit with the Exit icon after the calibration ends. 
4. Perform a delay calibration.

1. Connect the turbine to the optoelectronic reader and connect the sampling tube. Ensure that both are connected to the spirometer.

2. Synchronize breathing with the acoustic signal. This can be performed by the operator. NOTE: This procedure needs to be repeated every time a sampling tube is changed. Clean or switch the mask used for this procedure before giving it to the participant.

3. Exit with the Exit icon after the calibration ends.

3. Put the spirometer mask on the participant. Adjust the elastic bands on the head cap to create a tight seal around the face of the subject. NOTE: Optionally connect a heart-rate monitor to the spirometer and let the participant wear the heart-rate belt.

4. Fixate the hose of the spirometer so it does not interfere with movement.

5. Press Test, then enter a New subject on the display of the spirometer.

6. For submaximal exercise testing choose the breath-by-breath mode. To start recording press the Record key on the spirometer. NOTE: Scripts for the analysis of spirometer data are available in the Supplementary Material 6.

\section{Testing procedure}

1. Instruct the participant to perform 4 min of steady-state exercise at the desired speed $(1.11 \mathrm{~m} / \mathrm{s})$

1. Instruct the participant to use velocity feedback to stay (on average) at the desired speed.

NOTE: Speed can be shown from the measurement wheel or the IMUs from their respective laptops in the overground condition. The laptops have hook-and-loop straps that allow for fixation on the legs.

2. Instruct the participant to stay (on average) in the center of the treadmill for the treadmill condition.

3. Instruct the participant to look at the velocity and heading feedback on the ergometer screen in the ergometer condition and keep it (on average) within the target range.

2. Start a stopwatch and the spirometer (step 5.6) at the same time.

NOTE: This can be done without an external trigger as the timing difference from pressing start is negligible while using breath-by-breath spirometry.

3. After $30 \mathrm{~s}$, start the wheelchair propulsion.

NOTE: For the treadmill and ergometer conditions this implies starting the treadmill or ergometer. When using a measurement wheel (step 2.4.1.8) or IMUs (step 2.4.2.3) start those as well.

1. Use the lap button in the overground condition to mark the corners of the track.

4. After another 4 min during the test, without prior notice, instruct the participant to stop pushing the wheelchair. NOTE: In the treadmill condition a few additional pushes are needed before the belt stops.

\section{Representative Results}

Using the aforementioned procedure, power output was determined for 17 familiarized (two 30 min sessions of practice) able-bodied participants with an overground back-and-forth coast-down test (mean of five trials). The coast-down profile was characterized with a measurement wheel in a smooth hospital hallway. Afterwards, participants were measured during overground $(25.0 \times 9.0 \mathrm{~m}$ circuit), treadmill $(2.0 \times 1.2 \mathrm{~m})$, and ergometer wheelchair propulsion. The power output in the treadmill and ergometer modalities were matched with the overground condition using the protocols described in this paper.

Power output was obtained from the same measurement wheel during three blocks of 4 min of wheelchair propulsion after a familiarization block of equal length. Only the last minute of each block was used for analysis, assuming steady-state propulsion. For the overground propulsion data only the long straights $(25 \mathrm{~m}$ ) were used. All data (pre-)processing was performed in Python 3.7 (Python Software Foundation). ICC estimates and their 95\% confidence intervals were calculated in R 3.3.4 (R Core Team), using a single-rating, absolute-agreement, random-effects model.

The mean combined weight of the wheelchair-user system was $92.6 \mathrm{~kg}( \pm 8.3)$. The mean expected power output from the coast-down test was $9.7 \mathrm{~W}( \pm 1.6)$. Power output as calculated from the measurement wheel was lower for overground $8.1 \mathrm{~W}( \pm 1.4)$, treadmill $7.8 \mathrm{~W}( \pm 1.9)$, and ergometer $8.7 \mathrm{~W}( \pm 2.2)$ wheelchair propulsion. The average difference between target power output and measured power output were -1.6 $( \pm 1.6),-1.8( \pm 1.4),-1.0( \pm 1.0) \mathrm{W}$ for overground, treadmill, and ergometer propulsion, respectively. These results are also shown in Table 1 , Figure 5, and Figure 6.

Power output for overground propulsion showed a poor-to-moderate (ICC: $0.38, \mathrm{Cl}$ : 0.00-0.73) agreement with the target output. In contrast, treadmill propulsion showed poor-to-good (ICC: 0.45 , Cl: 0.00-0.79) agreement and ergometer propulsion showed poor-to-excellent (ICC: 0.77 , $\mathrm{Cl}$ : 0.11-0.93) agreement. Absolute error was negatively correlated with power output for propulsion on the ergometer $(r=-0.55, p=0.02)$, but not for the other two conditions (overground: $r=0.47, p=0.06$; treadmill: $r=0.22, p=0.40$ ).

Agreement between conditions was poor-to-moderate (ICC: $0.49, \mathrm{CI}: 0.20-0.74)$. Within-modality (between the three 4 min blocks) reliability was good-to-excellent for overground (ICC: 0.91, Cl: 0.82-0.97) and treadmill (ICC: 0.97, Cl: 0.93-0.99) propulsion and moderate-to-excellent for ergometer propulsion (ICC: $0.97, \mathrm{Cl}: 0.71-0.99)$. The ergometer appeared to perform worse over time, which was confirmed by a repeatedmeasures ANOVA $(F(2,32)=64.7, p<0.01)$, but there was no time effect for overground $(F(2,32)=0.9, p=0.418)$ and treadmill $(F(2,32)=$ $0.9, p=0.402)$ propulsion. 


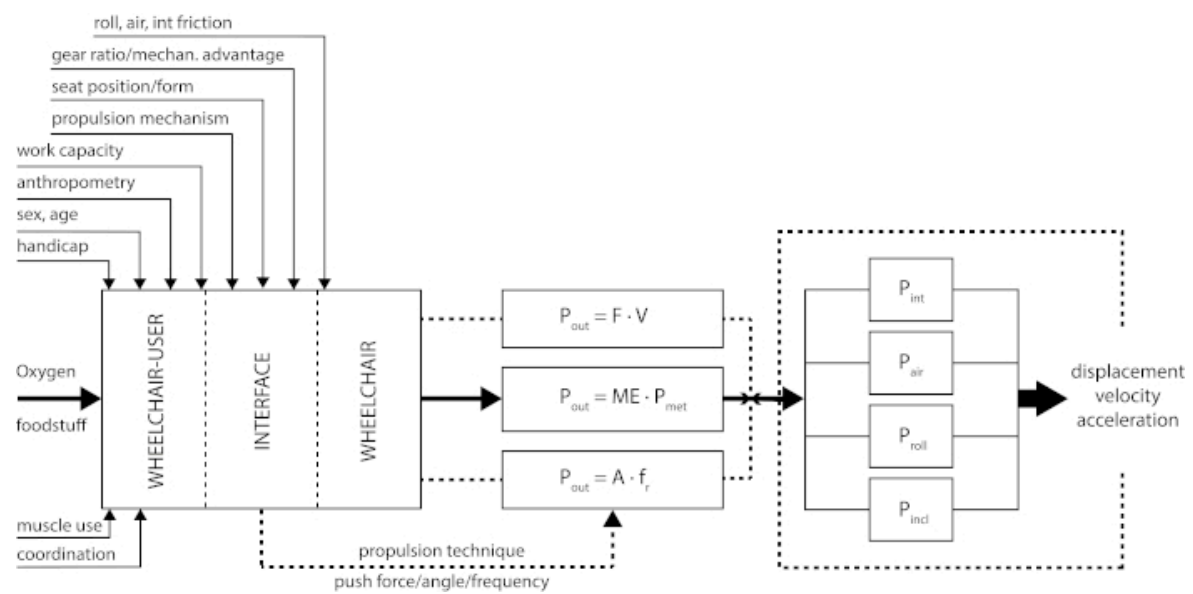

Figure 1: Power balance applied to manual wheelchair propulsion. $P_{\text {out }}$ external power output (W); ME: gross mechanical efficiency (\%); $F$ : mean resisting force; V: mean coasting velocity; A: work per push or cycle $(J)$; $f_{r}$ : frequency of pushes or cycles $(1 / s)$; $P_{\text {int }}$ : internal losses $(W)$; $\mathrm{P}_{\text {air }}$ : aerodynamic resistance $(\mathrm{W}) ; \mathrm{P}_{\text {roll }}$ : rolling friction $(\mathrm{W}) ; \mathrm{P}_{\text {incl }}$ : losses due to inclination $(\mathrm{W})$. This figure is reprinted from van der Woude et al. ${ }^{20}$. Please click here to view a larger version of this figure.

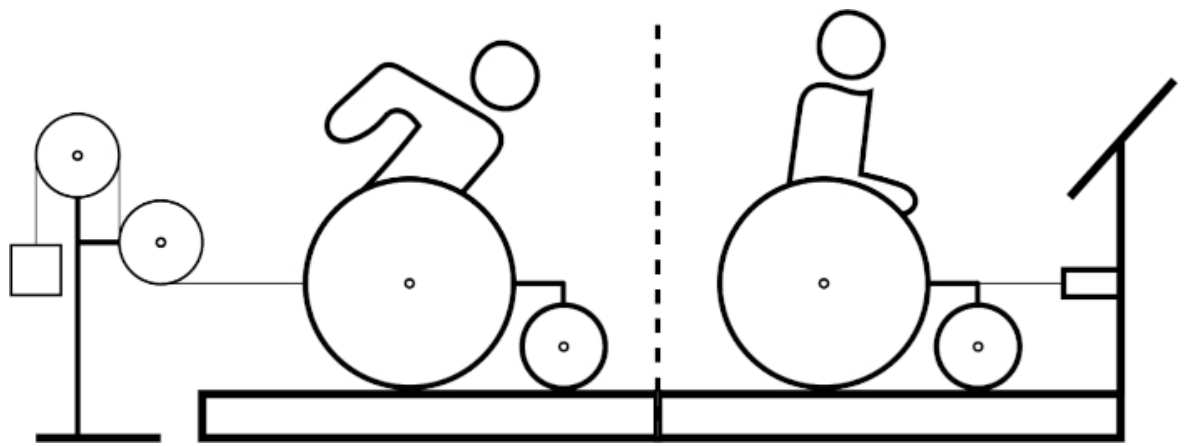

Figure 2: Treadmill setup. Left: Pulley setup to increase the external power output on a treadmill during propulsion. Right: Drag test setup to measure the frictional forces during treadmill wheelchair propulsion. Please click here to view a larger version of this figure. 


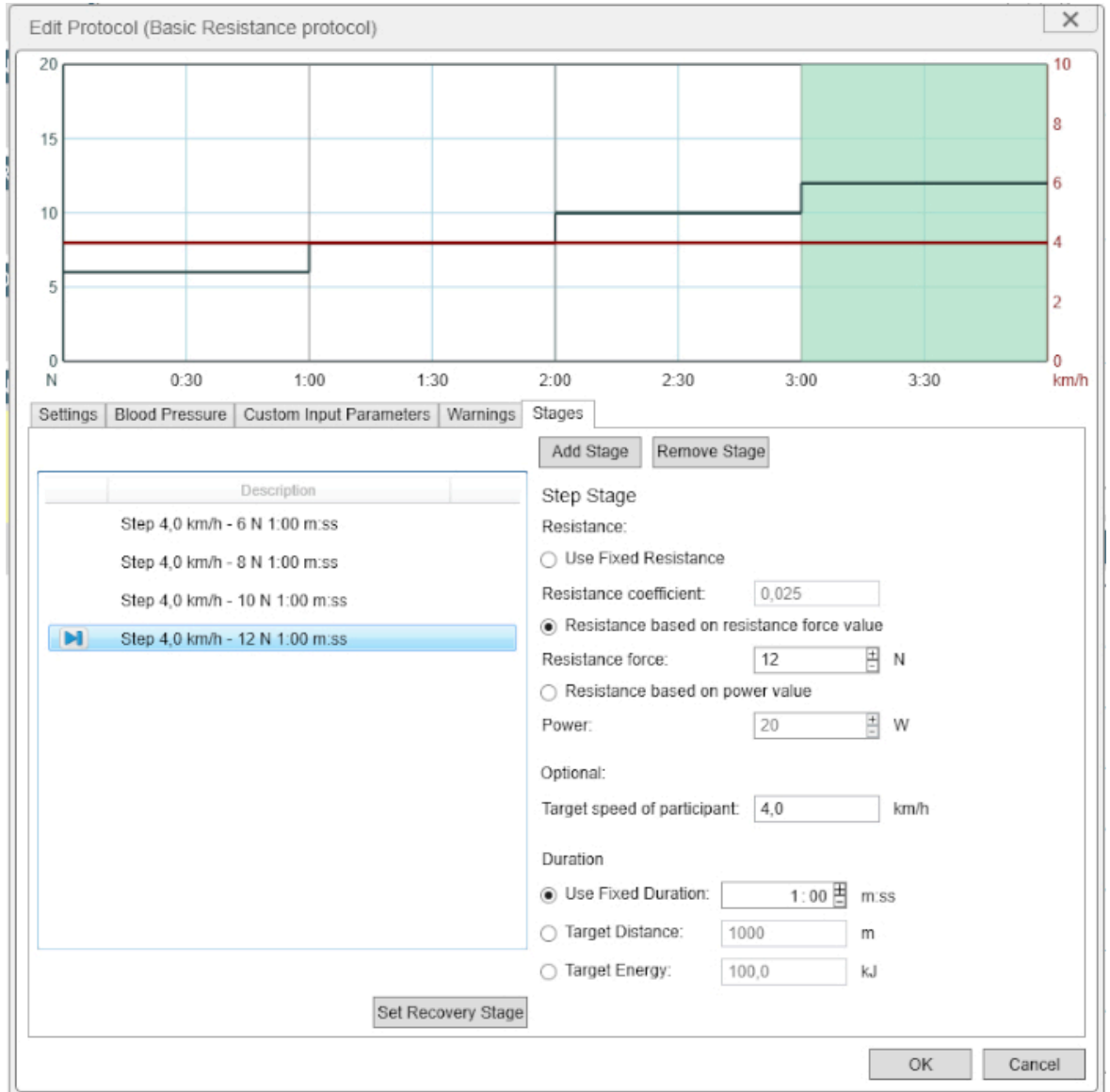

Figure 3: Protocol settings window for the wheelchair ergometer. Power output can be set by choosing a power output and a target speed or a rolling friction and a target speed. Please click here to view a larger version of this figure.

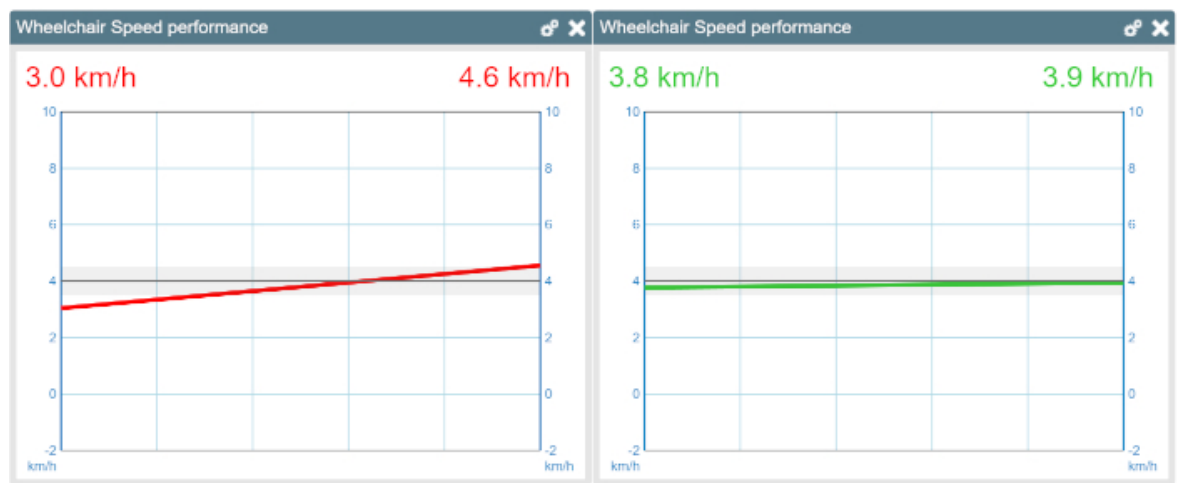

Figure 4: Feedback on the wheelchair ergometer in the form of a line plot. Left and right roller speeds are plotted. Participants should try to keep a steady speed while going in a straight line (by keeping the on-screen line horizontal). Speed data is smoothed with a sliding window that can be changed in the settings. Please click here to view a larger version of this figure. 

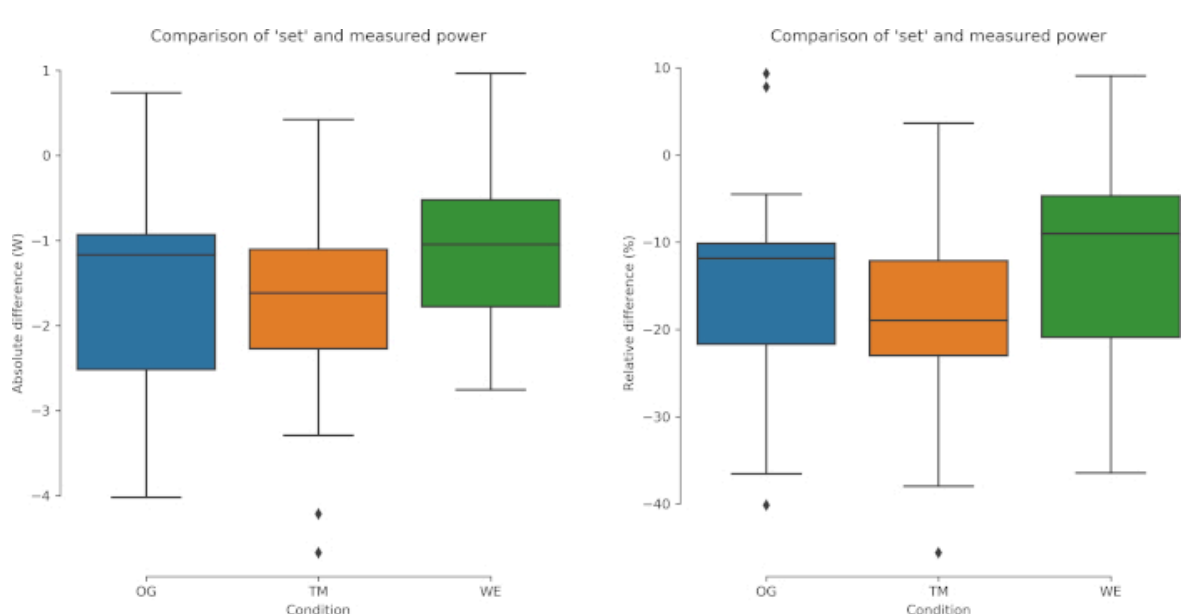

Figure 5: Relative and absolute difference distributions between coast-down friction and measured power output during overground (OG), treadmill (TM), and ergometer (WE) wheelchair propulsion. The whiskers show 1.5x the interquartile range. Please click here to view a larger version of this figure.
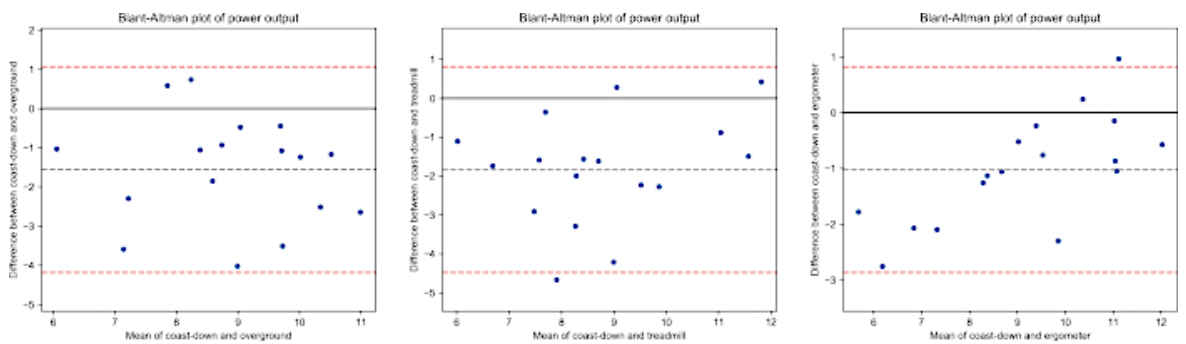

Figure 6: Bland-Altman plot for coast-down friction and measured power output during overground (left), treadmill (middle), and ergometer (right) wheelchair propulsion. The dark gray dotted lines indicate the pooled mean for a combination and the red dotted lines are the mean +1.96 standard deviations. Please click here to view a larger version of this figure.

\begin{tabular}{|l|l|l|l|l|l|l|}
\hline & $\begin{array}{l}\text { Value two-sided } \\
(\mathrm{W})^{2}\end{array}$ & $\begin{array}{l}\text { Difference with } \\
\text { target }\end{array}$ & $\begin{array}{l}\text { Difference with } \\
\text { target }(\%)\end{array}$ & $\begin{array}{l}\text { Difference with } \\
\text { target }(\mathrm{abs})\end{array}$ & $\begin{array}{l}\text { Agreement with } \\
\text { target PO (ICC) })^{3}\end{array}$ & $\begin{array}{l}\text { Reliability between } \\
\text { blocks }(\text { ICC })^{3}\end{array}$ \\
\hline Target $\mathrm{PO}^{1}$ & $9.68( \pm 1.57)$ & n.a & n.a & n.a. & n.a & n.a. \\
\hline Overground PO & $8.12( \pm 1.41)$ & $-1.56( \pm 1.57)$ & $-15.30( \pm 13.70)$ & $1.72( \pm 1.57)$ & $0.38(0.00-0.73)^{\star}$ & $0.91(0.82-0.97)^{\star}$ \\
\hline Treadmill PO & $7.84( \pm 1.92)$ & $-1.84( \pm 1.38)$ & $-18.98( \pm 13.42)$ & $1.91( \pm 1.16)$ & $0.45(0.00-0.79)^{\star}$ & $0.97(0.93-0.99)^{\star}$ \\
\hline Ergometer PO & $8.65( \pm 2.24)$ & $-1.02( \pm 0.97)$ & $-11.82( \pm 11.94)$ & $1.16( \pm 0.78)$ & $0.77(0.11-0.93)^{\star}$ & $0.97(0.71-0.99)^{\star}$ \\
\hline
\end{tabular}

1. Calculated from coast-down friction. 2. Determined with measurement wheel. 3. Two-way, absolute agreement, fixed raters with $95 \%$ confidence intervals. ${ }^{*} p<0.001$.

Table 1: Comparison of set power output and actual power output measured with a measurement wheel. 


\begin{tabular}{|l|l|}
\hline Factors & Rolling resistance \\
\hline Body mass $\uparrow$ & $\uparrow$ \\
\hline Wheelchair mass $\uparrow$ & $\uparrow$ \\
\hline Tire pressure $\downarrow$ & $\uparrow$ \\
\hline Wheel size $\uparrow$ & $\downarrow$ \\
\hline Hardness floor $\downarrow$ & $\uparrow$ \\
\hline Camber angle $\uparrow$ & $?$ \\
\hline Toe-in/out $\uparrow$ & $\uparrow \uparrow$ \\
\hline Castor shimmy $\uparrow$ & $\uparrow$ \\
\hline Center of mass on rear wheels & $\downarrow$ \\
\hline Folding frame & $\uparrow$ \\
\hline Maintenance $\downarrow$ & $\uparrow$ \\
\hline
\end{tabular}

Table 2: Factors influencing rolling friction and power output during manual wheelchair propulsion. This table is reprinted from van der Woude et al. ${ }^{8}$.

Supplementary Material 1. Please click here to view this file (Right click to download).

Supplementary Material 2. Please click here to view this file (Right click to download).

Supplementary Material 3. Please click here to view this file (Right click to download).

Supplementary Material 4. Please click here to view this file (Right click to download).

Supplementary Material 5. Please click here to view this file (Right click to download).

Supplementary Material 6. Please click here to view this file (Right click to download).

\section{Discussion}

In the previous sections an accessible methodology for determining and standardizing power output for different laboratory-based modalities was presented. Additionally, a comparison between set power output and measured power output during steady-state propulsion was made. While systematic error was present as well as some variability, the tools presented are better than the alternative: not standardizing at all. These results are similar to another study that reported measured power output and set power output ${ }^{50}$. Moreover, agreement between conditions was poor-to-moderate, indicating that extra attention should be paid when comparing studies using different modalities. As expected, the ergometer condition presented the easiest environment to standardize from the perspective of the operator. The ergometer performed better in the high friction settings. The blocks $(3 \times 4 \mathrm{~min})$ within one modality showed good-to-excellent and moderate-to-excellent agreement. Interestingly, the ergometer performed worse over time, possibly due to sensor drift. Therefore, it might be prudent to recalibrate the ergometer between every block. Note that these results are for low-intensity steady-state exercise and could differ for different protocols.

Minor mechanical or ergonomic changes in the wheelchair-user combination can have a large impact on experimental outcomes ${ }^{12,51}$. Material maintenance and a full awareness of vehicle mechanical principles are essential for performance outcomes and the validity of the experiment. The vehicle mechanics (e.g., mass, wheel sizes, tire type and pressure, alignment) and fit (e.g., fore-aft position, center of mass, mass, frontal plane) of wheelchair-user combination will determine rolling and air drag in combination with environmental conditions. The mass and the orientation of the center of mass will affect rolling drag with respect to the larger rear wheels and the smaller castor wheels in front. A summary of factors influencing rolling friction is presented in Table 2. Moreover, the wheelchair is often individualized. Apart from the intervention conditions (e.g., vehicle mechanics or interface) at each test, the wheelchair conditions must also be constant and its vehicle mechanics, including frame, seat, and tires should be checked. The tires need to be at a fixed pressure over tests and among individuals. Important checkpoints ${ }^{52}$ are possible friction points, rear wheel position, and potential changes in wheel alignment ${ }^{36,53,54,55}$.

Overground testing also requires ambulant technology for each of the indicators for cardiopulmonary strain, kinematics, or kinetics outcomes. This can be met, but the practicality of complex measurements is limited in a non-research environment. Coast-down tests are specific for the individual wheelchair-user combination and rolling surface. However, they are static, so they might not capture all the characteristics of the wheelchair-user combination ${ }^{56}$. They are especially sensitive to changes in the center of mass, which might explain the small differences between the coast-down test and the measured overground power output. These limitations are also found in the drag test and ergometer calibration, which also assume a static position of the wheelchair user.

The drag test measures the resisting forces of rolling and internal drag of each individual wheelchair-user combination. It is clearly sensitive to vehicle mechanics of the wheelchair, but also position and body orientation of the user. A standardized procedure is essential ${ }^{20,36}$, where at a constant belt speed, the user-wheelchair combination is pulled over the belt being connected to a unidimensional calibrated force transducer on the frame of the treadmill at a series of slope angles (Figure 2). A treadmill adaptor for load cells that can be adjusted to the height of the center axis of the wheelchair is required. Using linear regression analysis provides a static estimate of the mean drag force on the treadmill belt at zero inclination for a given wheelchair-user combination, which provides the mean external power output with the product of belt speed and drag force. The drag test is robust with regards to small differences in the execution of the test by different operators (e.g., position of the rope) ${ }^{37}$. 
Although sometimes assumed an apparently simple test, each of the testing elements of the drag test requires understanding of the underlying theory and training on all the details of the procedures ${ }^{8}$. Similar to the coast-down test, this test is especially sensitive to changes in center of mass. Moreover, the behavior and sensitivity of the strain gauge-based force transducers, their consistent calibration (i.e., precision of calibration weights, sequence of mounting $)^{20,36,37}$, as well as any of the procedures of the drag test that are sensitive to changes in speed or inclination angle of the treadmill all have to be considered. This means that the treadmill itself needs to be checked and calibrated as well ${ }^{37}$. Consistent awareness of such noise generating phenomena must be tracked and executed in day-to-day experimentation.

Precision of power output-based simulations and their outcomes are fully dependent on the standardization, practice, and training of those who conduct the experiments. Diversity of treadmills, ergometers, or any other electronically motor driven device can be an issue, as shown by De Groot et al. ${ }^{51}$. In exchange of population-based data, one should be aware of the potential role of such differences on the test outcomes. In any wheelchair experiment, a proper explanation of the testing conditions and open presentation of the actual values for speed, resistance, and power output should be presented for any subgroup or measurement condition.

In wheelchair experimentation, heterogeneity of the test sample is hard to escape from when focusing on the actual wheelchair users. Among those, people with a spinal cord injury are most frequently subject to research, because they tend to have a stable spinal cord lesion for the rest of their lives. Lesion level, completeness, gender, age, talent, and training status determine the heterogeneity of such study groups ${ }^{57}$. Increasing the number of participants through multicenter collaboration is an important way to circumvent this and increase the power of experimentation ${ }^{57}$, even in the early stages of rehabilitation ${ }^{10}$. This paper is hopefully a stepping-stone to a broad discussion on wheelchair experimenting in rehabilitation and adaptive sports communities that hopefully leads to international collaboration and knowledge exchange through the existing and new networks of researchers. Availability of adequate testing infrastructure allows consistent monitoring and evaluation of progress in clinical rehabilitation, adaptive sports, and beyond.

\section{Disclosures}

The authors have nothing to disclose.

\section{Acknowledgments}

The preparation of this manuscript was financially supported by a grant from Samenwerkingsverband Noord-Nederland (OPSNN0109) and was co-financed by the PPP-allowance of the Top consortia for Knowledge and Innovation of the Ministry of Economic Affairs.

\section{References}

1. Flemmer, C. L., Flemmer, R. C. A review of manual wheelchairs. Disability and Rehabilitation: Assistive Technology. 11 (3), $177-187$ (2016).

2. WHO. World Report on Disability 2011. WHO Press. Geneva, Switzerland (2011).

3. Liu, X., Liu, N., Zhou, M., Lu, Y., Li, F. Bibliometric analysis of global research on the rehabilitation of spinal cord injury in the past two decades. Therapeutics and Clinical Risk Management. 15, 1-14 (2019).

4. Coe, P. L. Aerodynamic characteristics of wheelchairs. NASA Technical Memorandum 80191. (1979).

5. Khoo, S., Li, C., Ansari, P. The Top 50 Most Cited Publications in Disability Sport: A Bibliometric Analysis. Perceptual and Motor Skills. 125 (3), 525-545 (2018).

6. Cooper, R. A. Wheelchair research progress, perspectives, and transformation. Journal of Rehabilitation Research \& Development. 49 (1), 1-5 (2012).

7. de Groot, S. et al. WHEEL-I: development of a wheelchair propulsion laboratory for rehabilitation. Journal of Rehabilitation Medicine. 46 (6), 493-503 (2014).

8. van der Woude, L. H., Veeger, H. E., Dallmeijer, A. J., Janssen, T. W., Rozendaal, L. A. Biomechanics and physiology in active manual wheelchair propulsion. Medical Engineering \& Physics. 23 (10), 713-733 (2001).

9. van der Woude, L. H., de Groot, S., Janssen, T. W. Manual wheelchairs: Research and innovation in rehabilitation, sports, daily life and health. Medical Engineering \& Physics. 28 (9), 905-915 (2006).

10. de Groot, S. et al. Course of gross mechanical efficiency in handrim wheelchair propulsion during rehabilitation of people with spinal cord injury: a prospective cohort study. Archives of Physical Medicine and Rehabilitation. 86 (7), 1452-1460 (2005).

11. van Koppenhagen, C. F. et al. Patterns of Changes in Wheelchair Exercise Capacity After Spinal Cord Injury. Archives of Physical Medicine and Rehabilitation. 94 (7), 1260-1267 (2013).

12. van der Woude, L. H. et al. Wheelchair racing: effects of rim diameter and speed on physiology and technique. Medicine \& Science in Sports \& Exercise. 20 (5), 492-500 (1988).

13. van der Woude, L. H. V. et al. Seat height: effects on submaximal handrim wheelchair performance during spinal cord injury rehabilitation. Journal of Rehabilitation Medicine. 41 (3), 143-149 (2009).

14. Veeger, H. E., Rozendaal, L. A., van der Helm, F. C. Load on the shoulder in low intensity wheelchair propulsion. Clinical Biomechanics. 17 (3), 211-218 (2002).

15. Veeger, H. E. J., Vanderwoude, L. H. V., Rozendal, R. H. Load on the upper extremity in manual wheelchair propulsion. Journal of Electromyography and Kinesiology. 1 (4), 270-280 (1991).

16. Arnet, U., van Drongelen, S., Scheel-Sailer, A., van der Woude, L. H., Veeger, D. H. Shoulder load during synchronous handcycling and handrim wheelchair propulsion in persons with paraplegia. Journal of Rehabilitation Medicine. 44 (3), 222-228 (2012).

17. Vegter, R., de Groot, S., Lamoth, C., Veeger, D., Van der Woude, L. Initial Skill Acquisition of Handrim Wheelchair Propulsion: A New Perspective. IEEE Transactions on Neural Systems and Rehabilitation Engineering. (2013).

18. Vegter, R. J., Lamoth, C. J., de Groot, S., Veeger, D. H., van der Woude, L. H. Inter-individual differences in the initial 80 minutes of motor learning of handrim wheelchair propulsion. PLoS One. 9 (2), e89729 (2014).

19. van Ingen Schenau, G. J. Cycle power: a predictive model. Endeavour, New Series. 12 (1988). 
20. van der Woude, L. H., de Groot, G., Hollander, A. P., van Ingen Schenau, G. J., Rozendal, R. H. Wheelchair ergonomics and physiological testing of prototypes. Ergonomics. 29 (12), 1561-1573 (1986).

21. Vegter, R. J. K., de Groot, S., Hettinga, F. J., Veeger, H. E. J., van der Woude, L. H. V. Design of Manually Propelled Wheelchairs: Optimizing a Wheelchair-User Combination. http://cirrie.buffalo.edu (2010).

22. Janssen, T. et al. Relationship between physical strain during standardised ADL tasks and physical capacity in men with spinal cord injuries. Spinal Cord. 32 (12), 844 (1994).

23. de Klerk, R., Lutjeboer, T., Vegter, R. J. K., van der Woude, L. H. V. Practice-based skill acquisition of pushrim-activated power-assisted wheelchair propulsion versus regular handrim propulsion in novices. Journal of Neuroengineering and Rehabilitation. 15 (1), 56 (2018).

24. Vanderwoude, L. H. V. et al. Manual wheelchair propulsion-Effects of power output on physiology and technique. Medicine \& Science in Sports \& Exercise. 20 (1), 70-78 (1988).

25. Hintzy, F., Tordi, N. Mechanical efficiency during hand-rim wheelchair propulsion: effects of base-line subtraction and power output. Clinical Biomechanics. 19 (4), 343-349 (2004).

26. Chénier, F., Champagne, A., Desroches, G., Gagnon, D. H. Unmatched speed perceptions between overground and treadmill manual wheelchair propulsion in long-term manual wheelchair users. Gait \& Posture. 61 398-402 (2018).

27. Broucha, L., Krobath, H. Continuous recording of cardiac and respiratory functions in normal and handicapped people. Human Factors. 9 (6), 567-572 (1967).

28. Clarke, K. Caloric costs of activity in paraplegic persons. Archives of Physical Medicine and Rehabilitation. 47, 427-435 (1966).

29. Guo, L., Kwarciak, A. M., Rodriguez, R., Sarkar, N., Richter, W. M. Validation of a biofeedback system for wheelchair propulsion training. Rehabilitation Research and Practice. 2011, (2011).

30. Cooper, R. A. SMARTWheel: From concept to clinical practice. Prosthetics and Orthotics International. 33 (3), 198-209 (2009).

31. DiGiovine, C., Cooper, R., Dvornak, M. 'Magnificent Milestones and Emerging Opportunities in Medical Engineering' (Cat. No. 97CH36136). in Proceedings of the 19th Annual International Conference of the IEEE Engineering in Medicine and Biology Society. $1888-1891$ IEEE, (1997).

32. Theisen, D., Francaux, M., Fay, A., Sturbois, X. A new procedure to determine external power output during handrim wheelchair propulsion on a roller ergometer: a reliability study. International Journal of Sports Medicine. 17 (08), 564-571 (1996).

33. de Klerk, R. et al. Measuring handrim wheelchair propulsion in the lab: a critical analysis of stationary ergometers. IEEE Reviews in Biomedical Engineering. In press (2019).

34. van Ingen Schenau, G. J. Some fundamental aspects of the biomechanics of overground versus treadmill locomotion. Medicine \& Science in Sports \& Exercise. 12 (4), 257-261 (1980).

35. Voigt, E. D., Bahn, D. Metabolism and pulse rate in physically handicapped when propelling a wheel chair up and incline. Scandinavian Journal of Rehabilitation Medicine. 1 (3), 101-106. (1969).

36. Bennedik, K., Engel, P., Hildebrandt, G. Der Rollstuhl. Schindele Verlag (1978).

37. de Groot, S., Zuidgeest, M., van der Woude, L. H. Standardization of measuring power output during wheelchair propulsion on a treadmill Pitfalls in a multi-center study. Medical Engineering \& Physics. 28 (6), 604-612 (2006).

38. Veeger, H. E., van der Woude, L. H., Rozendal, R. H. Wheelchair propulsion technique at different speeds. Scandinavian Journal of Rehabilitation Medicine. 21 (4), 197-203 (1989).

39. Brattgard, S.-O., Grimby, G., Hook, O. Energy expenditure and heart rate in driving a wheelchair ergometer. Scandinavian Journal of Rehabilitation Medicine. 2, 143-148 (1970).

40. Niesing, R. et al. Computer-controlled wheelchair ergometer. Medical \& Biological Engineering \& Computing. 28 (4), $329-338$ (1990).

41. van der Woude, L. H., Dallmeijer, A. J., Janssen, T. W., Veeger, D. Alternative modes of manual wheelchair ambulation: an overview. American Journal of Physical Medicine \& Rehabilitation. 80 (10), 765-777 (2001).

42. Thomas, S., Reading, J., Shephard, R. J. Revision of the Physical Activity Readiness Questionnaire (PAR-Q). Canadian Journal of Sport Sciences. 17 (4), 338-345 (1992).

43. Chisholm, D. et al. PAR-Q validation report: the evaluation of a self-administered pre-exercise screening questionnaire for adults. Victoria: Canada: BC Ministry of Health and Health and Welfare. (1978).

44. Poole, D. C., Jones, A. M. Oxygen uptake kinetics. Comprehensive Physiology. 2 (2), 933-996 (2011).

45. Whipp, B. J., Wasserman, K. Oxygen uptake kinetics for various intensities of constant-load work. Journal of Applied Physiology. 33 (3), 351-356 (1972).

46. Veeger, H. E., van der Woude, L. H., Rozendal, R. H. Within-cycle characteristics of the wheelchair push in sprinting on a wheelchair ergometer. Medicine \& Science in Sports \& Exercise. 23 (2), 264-271 (1991).

47. van der Scheer, J. W., de Groot, S., Vegter, R. J., Veeger, D. H., van der Woude, L. H. Can a 15m-overground wheelchair sprint be used to assess wheelchair-specific anaerobic work capacity? Medical Engineering \& Physics. 36 (4), 432-438 (2014).

48. Van der Woude, L., Van Croonenborg, J., Wolff, I., Dallmeijer, A., Hollander, A. Physical work capacity after 7 wk of wheelchair training: effect of intensity in able-bodied subjects. Medicine \& Science in Sports \& Exercise. 31 (2), 331-341 (1999).

49. Fuss, F. K. Influence of mass on the speed of wheelchair racing. Sports Engineering. 12 (1), 41-53 (2009).

50. Vegter, R. J., Lamoth, C. J., De Groot, S., Veeger, D. H., Van der Woude, L. H. Variability in bimanual wheelchair propulsion: consistency of two instrumented wheels during handrim wheelchair propulsion on a motor driven treadmill. Journal of Neuroengineering and Rehabilitation. 10 (1), 9 (2013).

51. de Groot, S., Vegter, R. J., van der Woude, L. H. Effect of wheelchair mass, tire type and tire pressure on physical strain and wheelchair propulsion technique. Medical Engineering \& Physics. 35 (10), 1476-1482 (2013).

52. Khasnabis, C., Mines, K., Organization, W. H. Wheelchair service training package: basic level. World Health Organization. (2012).

53. Frank, T., Abel, E. Drag forces in wheelchairs. In Ergonomics of Manual Wheelchair Propulsion: State of the Art. Concerted Action 'Mobility Restoration for Paralyzed Persons'. Edited by Van der Woude, L. H. V., Meijs, P. J. M., Van der Grinten, B. A., De Boer, Y. A., 255-267, IOS Press. Amsterdam, Netherlands (1993).

54. Kauzlarich, J. Wheelchair rolling resistance and tire design. In Biomedical Aspects of Manual Wheelchair Propulsion: The State of the Art IIIAssistive Technology Research Series. Edited by Van der Woude, L. H. V., Hopman, M. T. E., Van Kemenda, C. H., 158-172, IOS Press. Amsterdam, Netherlands (1999).

55. Brubaker, C. E., McLaurin, C. A. Ergonomics of wheelchair propulsion. In Wheelchair III: report of a wheelchair on specially adapted wheelchairs and sports wheelchairs. 22-37 (1982). 
56. Eydieux, N. et al. Changes in wheelchair biomechanics within the first 120 minutes of practice: spatiotemporal parameters, handrim forces, motor force, rolling resistance and fore-aft stability. Disability and Rehabilitation: Assistive Technology. 1-9 (2019).

57. de Groot, S. et al. Demographics of the Dutch multicenter prospective cohort study 'Restoration of mobility in spinal cord injury rehabilitation'. Spinal Cord. 44 (11), 668-675 (2006). 